\title{
The Kinetics of Plastic Flow
}

\author{
B. Faucher and A. S. Krausz \\ Department of Mechanical Engineering, University of Ottawa, Ottawa, Canada
}

Z. Naturforsch. 35a, 1152-1161 (1980); received June 26, 1980

\begin{abstract}
The plastic flow associated with the three main methods of combining deformation mechanisms is described in terms of deformation kinetics. It is shown that the determination of the deformation kinetics as the initial step in the analysis is indispensable to the establishment of the activation parameters in functional forms. To this purpose, the effects of stress and temperature on the plastic strain rate are determined for three types of combined mechanisms, and qualitative comparison with experimental results is presented.
\end{abstract}

\section{Introduction}

Plastic flow within the usual stress and temperature ranges is a thermally activated process that is described generally by an Arrhenius-type equation as

$$
\dot{\varepsilon}_{\mathrm{p}}=\dot{\varepsilon}_{0} \exp (-\Delta G / k T)
$$

where $\dot{\varepsilon}_{\mathrm{p}}$ is the plastic strain rate, $\dot{\varepsilon}_{0}$ is the preexponential factor, $k$ is the Boltzmann constant, and $T$ is the absolute temperature. The Gibbs free energy of activation, $\Delta G$, is a function of the effective stress $\tau$ expressed as [1, 2]

$$
\Delta G(\tau)=\Delta G_{0}-\int_{0}^{\tau} V \mathrm{~d} \tau
$$

where $V$ is the activation volume.

Experimental results are often described in terms of (1), representing the effect of the stress and temperature by the empirical functions

$$
\Delta G=\Delta G(\tau, T), \quad V=V(\tau, T),
$$

and

$$
\dot{\varepsilon}_{0}=\dot{\varepsilon}_{0}(\tau, T) .
$$

These relations are concise and, therefore, conveniently represent the experimental behavior, but not necessarily provide physically correct descriptions of the deformation processes. Numerous relations based on micromechanical models have been proposed to describe plastic flow according to $(2)$; these models allow the determination of a "force-distance" relation that exists between, for example, a moving dislocation and the solute

Reprint requests to Dr. A.S. Krausz, Department of Mechanical Engineering, University of Ottawa, Ottawa, Ontario, KlN 6N5, Canada. atoms. Although these concepts are useful and certainly valid for a single dislocation, their direct application to experimental results through (1) is questionable. Similarly, the determination of a force-distance relation from experimental results is a mere rationalization of the observed behavior.

A more obvious drawback of the use of (2) with the Arrhenius relation is the limited validity of the latter: it is physically valid only when a single energy barrier controls the plastic flow and the backward activation is negligible, that is, at high stress level or low temperature. Even in these conditions, Eq. (2) remove the physical meaning of the Arrhenius equation that describes the effect of the temperature through the explicit temperature term in the exponential. This does not mean that $\Delta G$ and $\dot{\varepsilon}_{0}$ are temperature independent $(\Delta G$ is proportional to the elastic constants that are temperature dependent) but that this temperature dependence is negligible with respect to the explicit temperature term. Similarly, the stress dependence of the plastic strain rate has to follow essentially the stress dependence of the activation energy that is, in turn, affected by the work done during the activation process.

Ashby [3] has clearly shown that it is only in limited stress and temperature ranges that a single energy barrier controls the plastic flow. Since generally several processes are operating $[4,5]$, the parameters obtained from Eq. (1) are only phenom. enological parameters, weighted averages, and do not reflect the properties of a single mechanism [6]. To obtain a physically meaningful description of the experimental results, a kinetics analysis $[7,8]$ has to be carried out first, that will give the appropriate combination of the mechanisms. Then, as 
it is practiced in chemical kinetics studies, the combination of the individual rate constants can be determined [9-11]. Finally, from the kinetics equation the functional form of $\Delta G, V$ and $\dot{\varepsilon}_{0}$ may be derived.

In this study, combinations of the elementary processes are presented in a comprehensive and rigorous manner. The stress and temperature dependence of the strain rate obtained for each combination may serve as a guide for the determination of the kinetics associated with the behavior in the experimental results.

\section{The Combinations of the Elementary Processes}

(i) The movement of dislocations along various slip planes $[4,5]$, or the simultaneous operation of various types of flow units (dislocations, vacancies) that produce the observed strain rate are controlled by mechanisms that may be designated as "parallel-independent" ("PI").

(ii) "Parallel-dependent" ("PD") association of mechanisms was one of the first combinations utilized to describe the creep behavior of many materials [12]. This association corresponds to the movement of dislocations that encounter obstacles in their slip plane, such as the combined resistance raised by the forest dislocations and various types of precipitates, or the Peierls-Nabarro energy barrier.

(iii) The rate controlling mechanisms are "consecutive" ("C") when the dislocation motion obstacles consist of energy barriers in series. This is the case when the dislocation movement results from double kink nucleation and sideways spreading, controlled by point defects $[6,13-15]$. This also happens in the movement of jogged screw dislocations because the displacement of the jogs requires vacancy diffusion [16].

\section{The Kinetics Description}

The plastic strain rate caused by dislocation movement is given as

$$
\dot{\varepsilon}_{\mathrm{p}}=\alpha b \varrho \bar{v}
$$

where $\alpha$ is a geometrical factor, $b$ is the Burger's vector and $\varrho$ is the dislocation density. The average velocity $\bar{v}$ may be expressed as

$$
\bar{v}=\imath k
$$

where $l$ is the average distance moved by a dislocation after activation, and $k$ is the net activation rate that is a function of the actual combination of mechanisms. When a single energy barrier controls the process, the forward rate of activation is expressed as $[8,9]$

$$
k_{\mathrm{f}}=\frac{k T}{h} \exp \left(-\frac{\Delta G_{\mathrm{f}}}{k T}\right) .
$$

Combining these equations, the plastic strain rate in the forward direction only becomes

$$
\begin{aligned}
\dot{\varepsilon}_{\mathrm{f}} & =\alpha b \varrho l \frac{k T}{h} \exp \left(-\frac{\Delta G_{\mathrm{f}}}{k T}\right) \\
& =\dot{\varepsilon}_{0} \exp \left(-\frac{\Delta G_{\mathrm{f}}}{k T}\right) .
\end{aligned}
$$

The net rate is the difference between forward and backward activations $[8,17]$ :

$$
\begin{aligned}
\dot{\varepsilon}_{\mathrm{p}}= & \dot{\varepsilon}_{0 \mathrm{f}} \exp \left(-\Delta G_{\mathrm{f}} / k T\right) \\
& -\dot{\varepsilon}_{0 \mathrm{~b}} \exp \left(-\Delta G_{\mathrm{b}} / k T\right) .
\end{aligned}
$$

where the subscripts $f$ and $b$ identify the direction of the flow. The Gibbs free energy of activation in the two directions is defined by the rate theory as

and

$$
\Delta G_{\mathrm{f}}=\Delta G_{\mathrm{f}}^{\neq}-W_{\mathrm{f}}(\tau)
$$

$$
\Delta G_{\mathrm{b}}=\Delta G_{\mathrm{b}}^{\neq}+W_{\mathrm{b}}(\tau)
$$

where $W$ is the work done by the stress during the activation process, and $\Delta G^{\neq}$is the height of the free energy barrier. When backward activation is negligible, Eq. (3) reduces to (1). At low stress level, however, the work terms are small and backward activation must be considered. In fact, at zero stress level, the plastic strain rates resulting from forward and backward activations are equal, and the net plastic strain rate is zero. Equation (3) was shown to describe very well the deformation behavior of a number of materials $[17,18]$. For symmetrical energy barriers, (3) reduces to

$$
\begin{aligned}
\dot{\varepsilon}_{\mathrm{p}}= & 2 \dot{\varepsilon}_{0} \exp \left(-\Delta G^{\mp} / k T\right) \\
& \cdot \sinh [W(\tau) / k T] .
\end{aligned}
$$

When the plastic strain rate is determined by a combination of mechanisms, each barrier associated with a single mechanism is individually rate controlling only in a limited range of stress and temperature. The associated activation param- 
eters cannot be measured outside this range, and the work terms in this short range of stress may be linearized as

$$
\begin{aligned}
\Delta G(\tau) & =\Delta G^{\neq}-W(\tau) \\
& =\Delta G\left(\tau_{0}\right)+\left(\tau-\tau_{0}\right) \frac{\mathrm{d} \Delta G}{\mathrm{~d} \tau}+\cdots .
\end{aligned}
$$

Utilizing the notations

$$
V=-\left.\frac{\mathrm{d} \Delta G}{\mathrm{~d} \tau}\right|_{\tau_{0}} ; \quad \Delta G_{0}=\Delta G\left(\tau_{0}\right)+V \tau_{0}
$$

where $\tau_{0}$ is any reference stress in the range where the energy barrier is individually rate controlling (Fig. 1), the Gibbs free energy is then expressed as

$$
\Delta G(\tau)=\Delta G_{0}-V \tau
$$

where the activation volume $V$ is considered to be constant.

For parallel-independent combinations, the plastic strain rate is the sum of the contributions from each mechanism. When the energy barriers are symmetrical, $\dot{\varepsilon}_{\mathrm{p}}$ is expressed as

$$
\begin{aligned}
\dot{\varepsilon}_{\mathrm{p}}= & \sum_{i} \dot{\varepsilon}_{i}=\sum_{i} 2 \dot{\varepsilon}_{0, i} \\
& \cdot \exp \left(-\Delta G_{0, i} / k T\right) \sinh \left(V_{i} \tau / k T\right)
\end{aligned}
$$

where the orientation of the slip planes is taken into consideration by $\dot{\varepsilon}_{0, i}[4,5]$. For parallel-dependent associations, when the dislocation line moves at

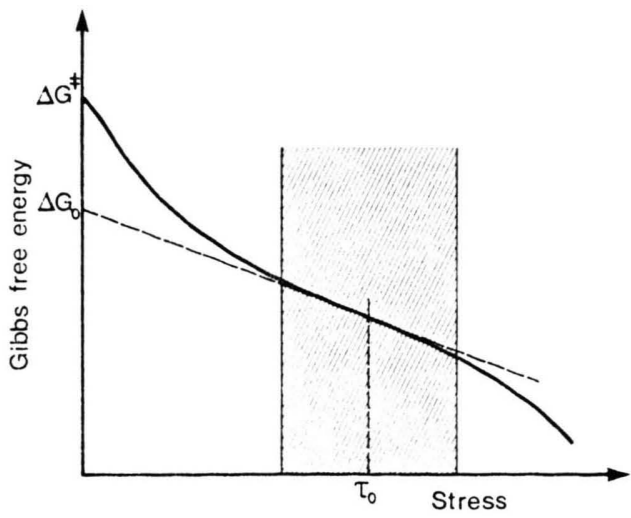

Fig. 1. Stress dependence of the activation energy for an energy barrier that controls the plastic strain rate individually only in the shaded stress range. The dashed line is the useful, observable approximation.

a uniform rate, the stress is the sum of the stresses associated with each of the individual barriers [19]:

$$
\tau=\sum_{i} \tau_{i}
$$

and for symmetrical barriers [7, 12]

$$
\tau=\sum_{i} \frac{k T}{V_{i}} \sinh ^{-1}\left[\frac{\dot{\varepsilon}}{2 \dot{\varepsilon}_{0, i}} \exp \left(\frac{\Delta G_{0, i}}{k T}\right)\right] .
$$

The behavior of a system of $n$ consecutive energy barriers is usually described by equating the flows $F_{i}$ (Fig. 2) over the $n$ barriers of the system $[8,20]$.

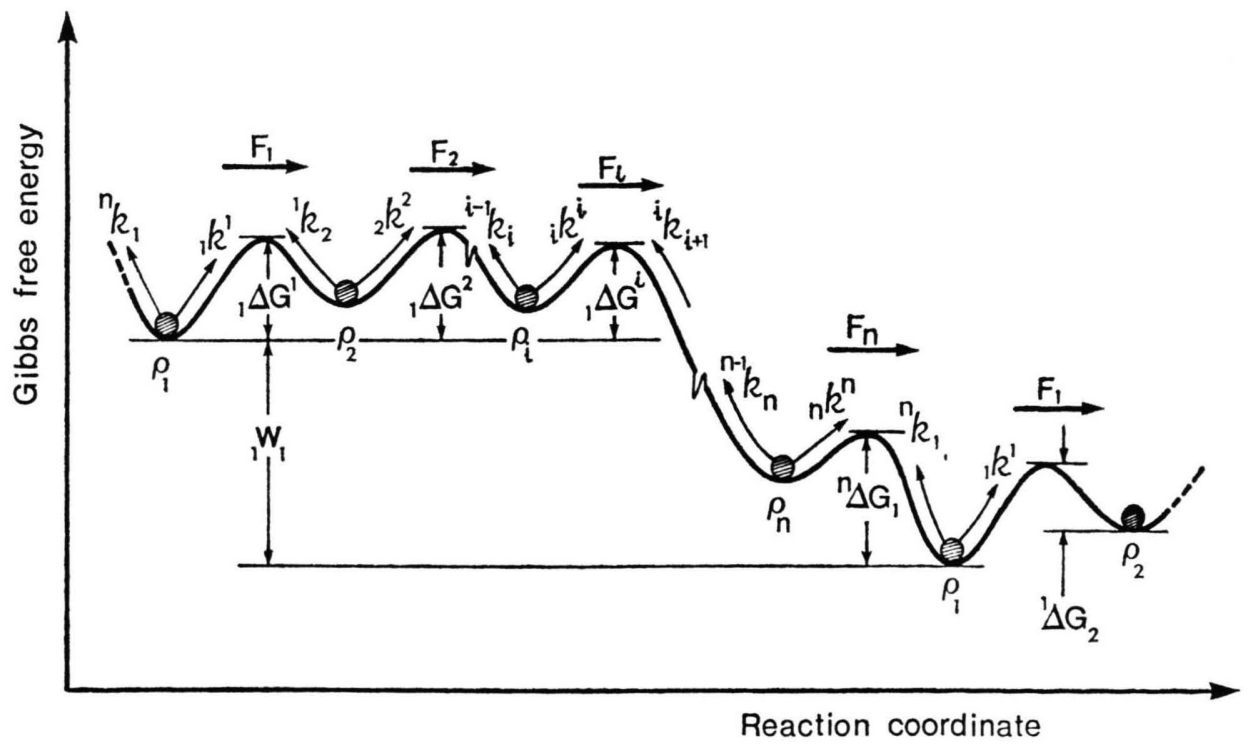

Fig. 2. Schematic representation of a system of $n$ consecutive energy barriers. 
It can be shown (see Appendix) that in steady-state the plastic strain rate is

$\dot{\varepsilon}_{\mathrm{p}}=\varepsilon_{0}\left[1-\exp \left(-{ }_{1} W_{1} / k T\right) / \sum_{i=1}^{n}\left(\sum_{j=i}^{i+n-1} t_{i j}\right)\right]$

and the dislocation densities in front of each barrier are

$$
\varrho_{i}=\varrho_{t} \sum_{j=i}^{n+i-1} t_{i j} / \sum_{i=1}^{n} \sum_{j=i}^{n+i-1} t_{i j}
$$

\section{The Stress Dependence of the Plastic Strain Rate}

The stress dependence of the plastic strain rate can be established from the rigorous kinetics descriptions given by Eqs. (5) to (7). The derivation will be carried out for two energy barriers, because the generalization is easy to obtain $[21-23]$. Accordingly, for the parallel-independent processes (5) becomes

$$
\begin{aligned}
\dot{\varepsilon}_{\mathrm{p}}= & \dot{\varepsilon}_{01} \exp \left(-\frac{\Delta G_{01}-V_{1} \tau}{k T}\right) \\
& +\dot{\varepsilon}_{02} \exp \left(-\frac{\Delta G_{02}-V_{2} \tau}{k T}\right)
\end{aligned}
$$

for the parallel-dependent mechanisms Eq. (6) is

$$
\begin{aligned}
\tau=\tau_{1}+\tau_{2}= & \frac{k T}{V_{1}} \sinh ^{-1}\left[\frac{\dot{\varepsilon}_{\mathrm{p}} \exp \left(\Delta G_{01} / k T\right)}{2 \dot{\varepsilon}_{01}}\right] \\
& +\frac{k T}{V_{2}} \sinh ^{-1}\left[\frac{\dot{\varepsilon}_{\mathrm{p}} \exp \left(\Delta G_{02} / k T\right)}{2 \dot{\varepsilon}_{02}}\right],
\end{aligned}
$$

and, in the case of two consecutive energy barriers, (7) takes the form

$$
\begin{aligned}
\dot{\varepsilon}_{\mathrm{p}}=\dot{\varepsilon}_{0} /[ & \exp \left(\frac{\Delta G_{01}-V_{1} \tau}{k T}\right) \\
& \left.+\exp \left(\frac{\Delta G_{02}-V_{2} \tau}{k T}\right)\right] .
\end{aligned}
$$

In (9) and (11), the terms associated with backward activation have been neglected; they will be considered only qualitatively at low stress level.

The stress dependence of the strain rate is shown in Fig. 3 for the three types of combination. The curves were calculated with the use of (9) to (11), by determining the behavior at low and high stress levels. For example, in the case of parallel-dependent mechanisms, when $\tau_{1}$ and $\tau_{2}$ are both large, the arguments of the $\sinh ^{-1}$ functions are large

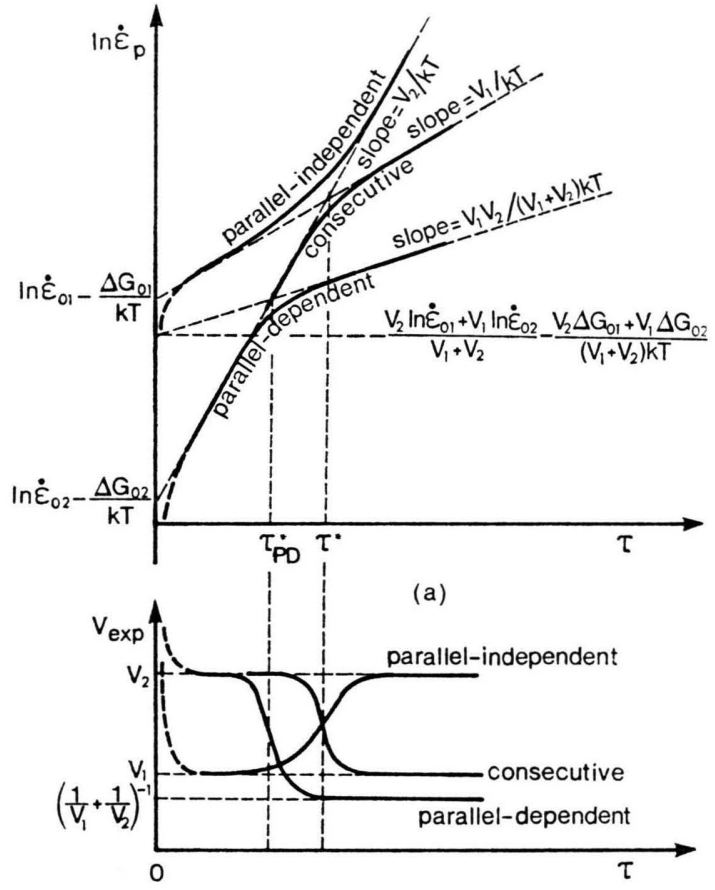

(b)

Fig. 3. Schematic stress dependence of (a) the plastic strain rate and (b) the experimental activation volume for the various combinations of two mechanisms.

and $(10)$ reduces to

$$
\begin{aligned}
\tau=\tau_{1}+\tau_{2}= & \frac{k T}{V_{1}} \ln \left[\frac{\dot{\varepsilon}_{\mathrm{p}} \exp \left(\Delta G_{01} / k T\right)}{\dot{\varepsilon}_{01}}\right] \\
& +\frac{k T}{V_{2}} \ln \left[\frac{\dot{\varepsilon}_{\mathrm{p}} \exp \left(\Delta G_{02} / k T\right)}{\dot{\varepsilon}_{02}}\right]
\end{aligned}
$$

whence

$$
\begin{aligned}
\ln \dot{\varepsilon}_{\mathrm{p}}= & \frac{V_{2} \ln \dot{\varepsilon}_{01}+V_{1} \ln \dot{\varepsilon}_{02}}{V_{1}+V_{2}} \\
& -\frac{V_{2} \Delta G_{01}+V_{1} \Delta G_{02}-V_{1} V_{2} \tau}{\left(V_{1}+V_{2}\right) k T} .
\end{aligned}
$$

At low strain rate $\tau_{1}$ becomes very small and the ratio $\dot{\varepsilon}_{\mathrm{p}} \exp \left(\Delta G_{01} / k T\right) / 2 \dot{\varepsilon}_{01}$ is much less than one. Therefore, (10) reduces to

$$
\tau \cong \tau_{2}=\frac{k T}{V_{2}} \sinh ^{-1}\left[\frac{\dot{\varepsilon}_{\mathrm{p}} \exp \left(\Delta G_{02} / k T\right)}{2 \dot{\varepsilon}_{02}}\right]
$$

and when $\tau_{2}$ is large enough

$$
\tau \cong \frac{\Delta G_{02}}{V_{2}}+\frac{k T}{V_{2}} \ln \left(\dot{\varepsilon}_{\mathrm{p}} / \dot{\varepsilon}_{02}\right)
$$


whence

$$
\ln \dot{\varepsilon}_{\mathrm{p}}=\ln \dot{\varepsilon}_{02}-\frac{\Delta G_{02}-V_{02} \tau}{k^{\prime}}
$$

Figure $3 \mathrm{~b}$ indicates the stress dependence of the experimental activation volume, $V_{\exp }$, obtained from the relation

$$
V_{\exp }=k T \partial \ln \dot{\varepsilon}_{\mathrm{p}} / \partial \tau
$$

The experimental activation volume becomes infinitely large at low stress level because all curves in Fig. 3 a approach asymptotically the ordinate. This is a geometrically general, inescapable condition, because $\dot{\varepsilon}_{\mathrm{p}}=0$ is at minus infinity on the In axis. It has been shown $[17,24]$ that $\dot{\varepsilon}_{\mathrm{p}}=0$ when forward and backward activations are equal. The waviness of the curves in Fig. $3 \mathrm{~b}$, due to the combinations of mechanisms, may be smaller than the experimental scatter and are not necessarily observed. Many measurements, however, do show a behavior similar to the theoretical curves, and examples are given in Figs. 4 and 5 .

For each of the three combinations a transition stress $\tau^{*}$ may be defined as the stress at which the strain rates, obtained at low and high stress levels, are equal $[22,23,25]$. From Figure 3 a, or from the corresponding equations derived from (9) to (11), the transition stresses are

$$
\begin{aligned}
\tau_{\mathrm{PI}}^{*} & =\frac{\Delta G_{02}-\Delta G_{01}}{V_{2}-V_{1}}+\frac{k T}{V_{2}-V_{1}} \ln \left(\frac{\dot{\varepsilon}_{01}}{\dot{\varepsilon}_{02}}\right), \\
\tau_{\mathrm{PD}}^{*} & =\frac{\Delta G_{02}-\Delta G_{01}}{V_{2}}+\frac{k T}{V_{2}} \ln \left(\frac{\dot{\varepsilon}_{01}}{\dot{\varepsilon}_{02}}\right),
\end{aligned}
$$

and

$$
\tau_{\mathrm{C}}^{*}=\frac{\Delta G_{02}-\Delta G_{01}}{V_{2}-V_{1}} .
$$

These relations indicate that the transition stress is temperature dependent when the mechanisms are parallel-independent and parallel-dependent. The temperature independence of the transition stress for the consecutive systems is in contradiction with the conclusions of previous analyses that were based on the essentially phenomenological "power law" relation [22, 25]. Although it is a convenient relation for some engineering applications, it may lead to incorrect conclusions. For example, the constant stress exponent, that is, the constant slope of the $\ln \dot{\varepsilon}_{\mathrm{p}} \mathrm{vs} . \ln \tau$ curve indicates that a single mechanism operates. The same results analyzed in terms of deformation kinetics and represented in the $\ln \dot{\varepsilon}_{\mathrm{p}}$ vs. $\tau$ system would indicate the operation of either consecutive or parallel-dependent mechanisms. This example illustrates the precautions that must be taken before establishing the deformation mechanisms. It is of interest to note that while only two terms were used in Eq. (11) to describe the behavior at the transition in a consecutive energy barrier system, a comprehensive study of two barrier systems [23] has shown that three rate constants in the denominator of Eq. (7) may be individually rate controlling and thus two transitions may be observed.

Figure 4 shows some of the stress relaxation results of Law and Beshers [26] obtained at room temperature for various metals. Comparison with Fig. 3 indicates that the plastic flow of fec metals may be controlled by parallel-independent mechanisms, that of iron either by consecutive or paralleldependent mechanisms, and that of titanium by a single energy barrier with constant activation volume. The stress dependence of the activation volume in high purity iron has been shown to be of the kind described in Fig. 5 [27-29]. It may be proposed that in region I of Fig. 5, a single mechanism controls the plastic flow and that the effect of backward activation is noticeable. In region II, the same mechanism is combined independently

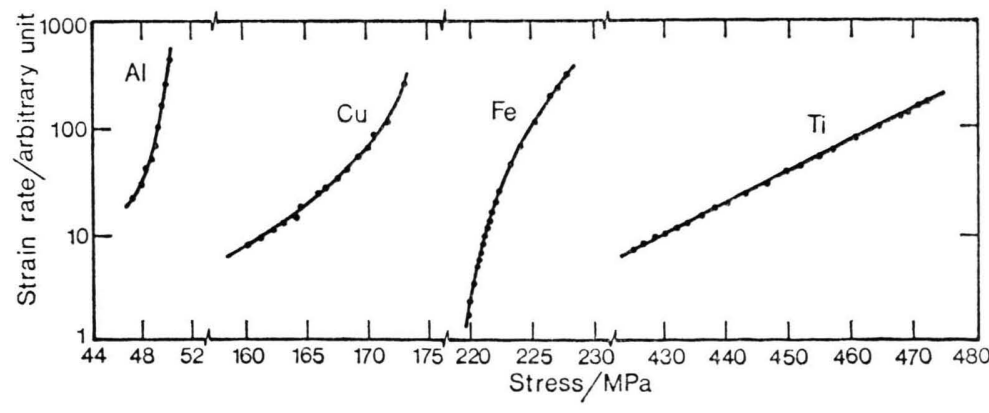

Fig. 4 .

Stress relaxation in metals at room temperature [26]. 


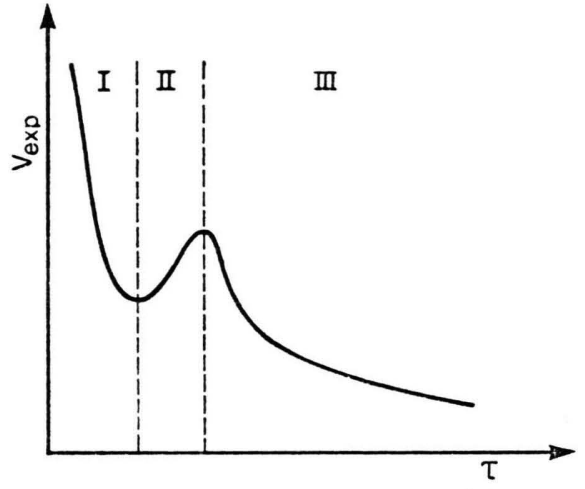

Fig. 5. Schematic representation of the stress dependence of the activation volume for plastic flow in pure iron [ $27-$ 29]. In Region I a single mechanism is operating, in region II two parallel-independent mechanisms, and in region III either two parallel-dependent or two consecutive mechanisms.

in parallel with a second mechanism, and in region III, there is either a parallel-dependent or a consecutive association of the second mechanism with a third one. The stress dependence of the three Gibbs free energies that could be associated with the three mechanisms is shown schematically in Figure 6. The activation volume for the third mechanism may be slightly stress dependent, particularly if it controls independently the rate of flow in a wide stress range. The behavior depicted in Fig. 5 usually disappears at higher carbon concentrations and the curve becomes monotonous. This may result from either an upward displacement of curve 1 in Fig. 6, or a displacement to the

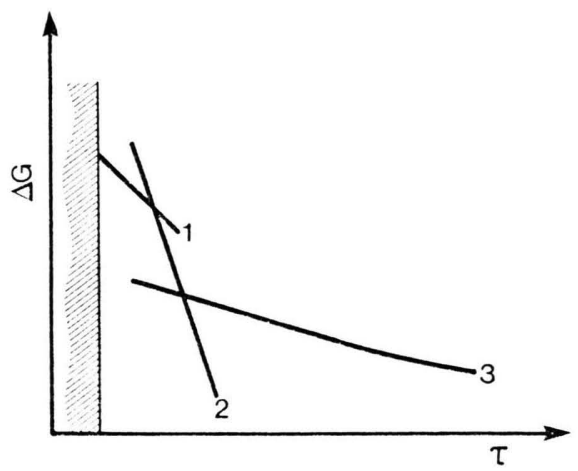

Fig. 6. Schematic stress dependence of the three Gibbs free energies that may be associated with the three mechanisms of Figure 5. Mechanism 1 is operating in region I, mechanisms 1 and 2 in region II and mechanisms 2 and 3 in region III. At low stress level (shaded region) backward activation becomes noticeable and should be considered. right of curve 2 due to changes in the free energies associated with changes in the carbon content. This tentative description of the experimental results is incomplete by far, nevertheless, it illustrates the possibilities of the kinetics analysis to improve our understanding of deformation behavior.

\section{The Temperature Dependence of the Plastic Strain Rate}

The temperature dependence of the strain rate is also obtained from Eqs. (9) to (11) by analyzing the behavior at low and high temperatures under constant stress. However, it can be deduced from the stress dependence also, because it is well-known that increasing the temperature has the same effect as decreasing the strain rate. For example, in the case of parallel-dependent mechanisms, the arguments of the $\sinh ^{-1}\left[\frac{\dot{\varepsilon}_{\mathrm{p}} \exp \left(\Delta G_{0 i} / k T\right)}{2 \dot{\varepsilon}_{0 i}}\right]$ functions are large at low temperature. The plastic strain rate at low temperature is thus described by Equation (12) that was used for high stress level. Similarly, Equation (13) describes the behavior of parallel-dependent mechanisms at both low stress level and high temperature. The temperature dependence of the strain rate is shown in Fig. 7 a for the various combinations. The experimental activation energies $\Delta G_{\text {exp }}$ were obtained from the empirical Arrhenius equation as

$$
\Delta G_{\exp }=-k \partial \ln \dot{\varepsilon}_{\mathrm{p}} / \partial 1 / T .
$$

It is of interest to note that for consecutive systems $\Delta G_{\exp }$ is almost independent of the temperature.

Similarly to the transition stress, a transition temperature $T^{*}$ may be defined as the temperature at which the strain rates, obtained at low and high temperatures, are equal. The same equations that were used to derive the stress dependence, Eqs. (12) and (13), can be used to derive the transition temperatures. Accordingly, the corresponding relations are

$$
T_{\mathrm{PI}}^{*}=\frac{\Delta G_{01}-\Delta G_{02}-\left(V_{1}-V_{2}\right) \tau}{k \ln \left(\dot{\varepsilon}_{01} / \dot{\varepsilon}_{02}\right)}
$$

and

$$
T_{\mathrm{PD}}^{*}=\frac{\Delta G_{01}-\Delta G_{02}+V_{2} \tau}{k \ln \left(\dot{\varepsilon}_{01} / \dot{\varepsilon}_{02}\right)} .
$$

For consecutive systems, because $\dot{\varepsilon}_{01} \equiv \dot{\varepsilon}_{02}$, the transition temperature becomes infinite, indepen- 


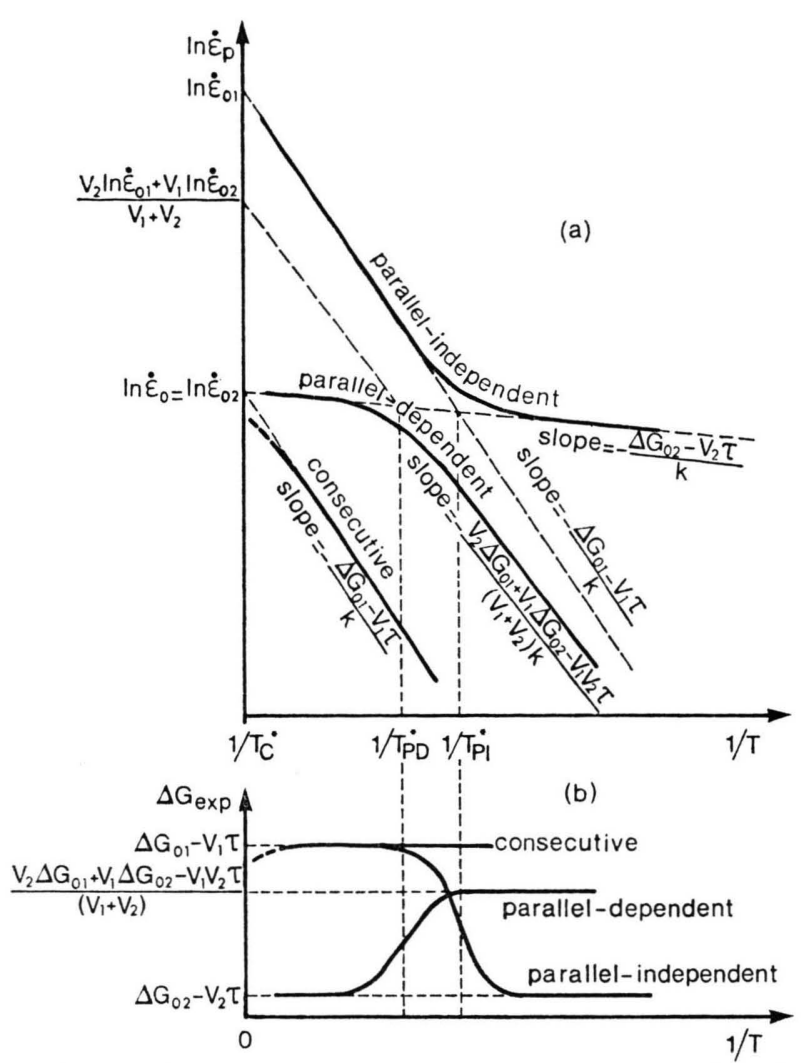

Fig. 7. Schematic temperature dependence of (a) the plastic strain rate and (b) the experimental activation energy for the various combination of two mechanisms.

dently of the stress level. This conclusion is again in contradiction with previous analyses; the reason is that, in general, the plastic strain rate for consecutive systems is obtained from the relation

$$
\frac{1}{\dot{\varepsilon}_{\mathrm{p}}}=\frac{1}{\dot{\varepsilon}_{1}}+\frac{1}{\dot{\varepsilon}_{2}}
$$

without specifying $\dot{\varepsilon}_{1}$ and $\dot{\varepsilon}_{2}$ (e.g. [22]). Deformation kinetics studies show (Appendix) that the plastic strain rate is

or

$$
\dot{\varepsilon}_{\mathrm{p}}=\varepsilon_{0} /\left(t_{1}+t_{2}\right)
$$

$$
\frac{1}{\dot{\varepsilon}_{\mathrm{p}}}=\frac{1}{\varepsilon_{0} / t_{1}}+\frac{1}{\varepsilon_{0} / t_{2}}
$$

where $t_{1}$ and $t_{2}$ are of the form

$$
t_{i}=\frac{h}{k T} \exp \left(\Delta G_{i} / k T\right) .
$$

The two strain rates $\dot{\varepsilon}_{1}$ and $\dot{\varepsilon}_{2}$ are, therefore, equal only at infinite temperature. If the temperature dependence of the Gibbs free energies is considered, the transition temperature for consecutive systems may be expressed as

$$
T_{\mathrm{C}}^{*}=\frac{\Delta H_{1}-\Delta H_{2}}{\Delta S_{1}-\Delta S_{2}}
$$

where $\Delta H$ and $\Delta S$ represent the enthalpy and the entropy of activation respectively. In general, it is assumed that the temperature dependence of the Gibbs free energy of activation is weak [1,30], therefore, both $\Delta S$ in (14) are small. Thus, the difference $\Delta S_{1}-\Delta S_{2}$ is very small and the transition temperature $T_{\mathrm{C}}^{*}$ very large.

The experimental results obtained by Burton and Reynolds [31] for high temperature creep of uranium dioxide are shown in Figure 8. The behavior is typical of consecutive or parallel-dependent combination of mechanisms. The analysis by Gilbert and Munson [32] of the experimental results obtained by several authors indicates that the

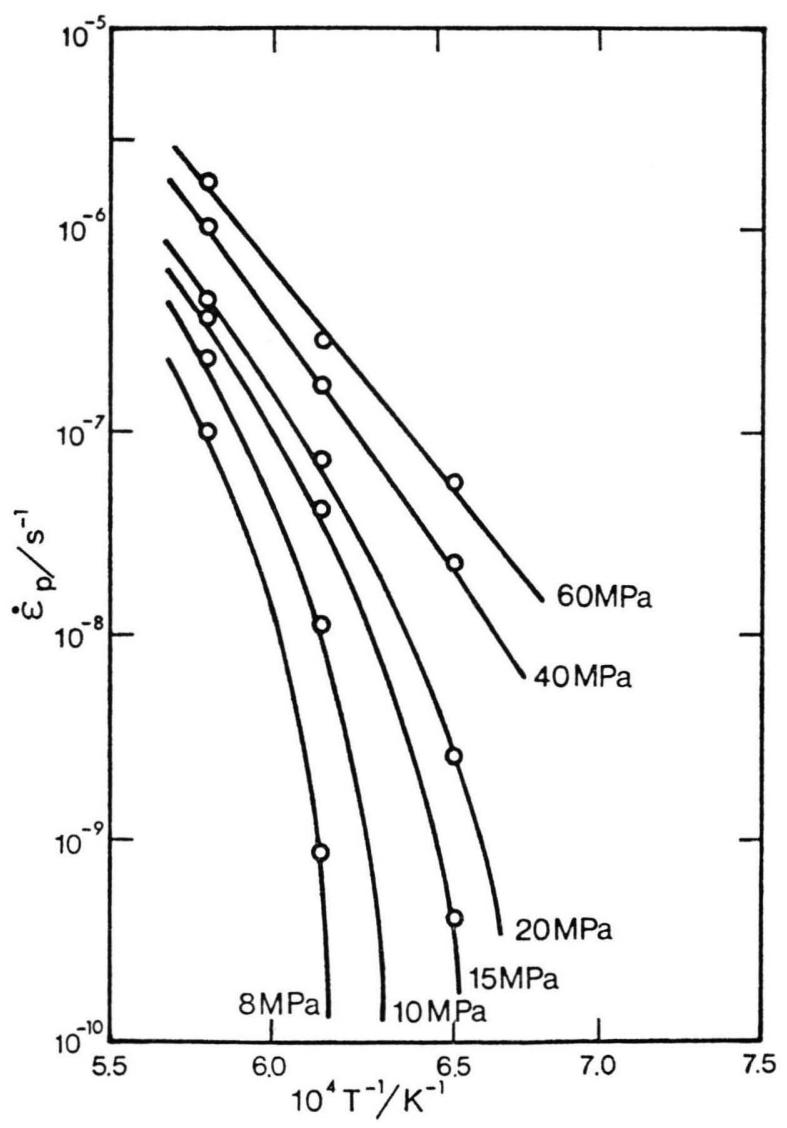

Fig. 8. The steady state creep rate of uranium dioxide [31]. 


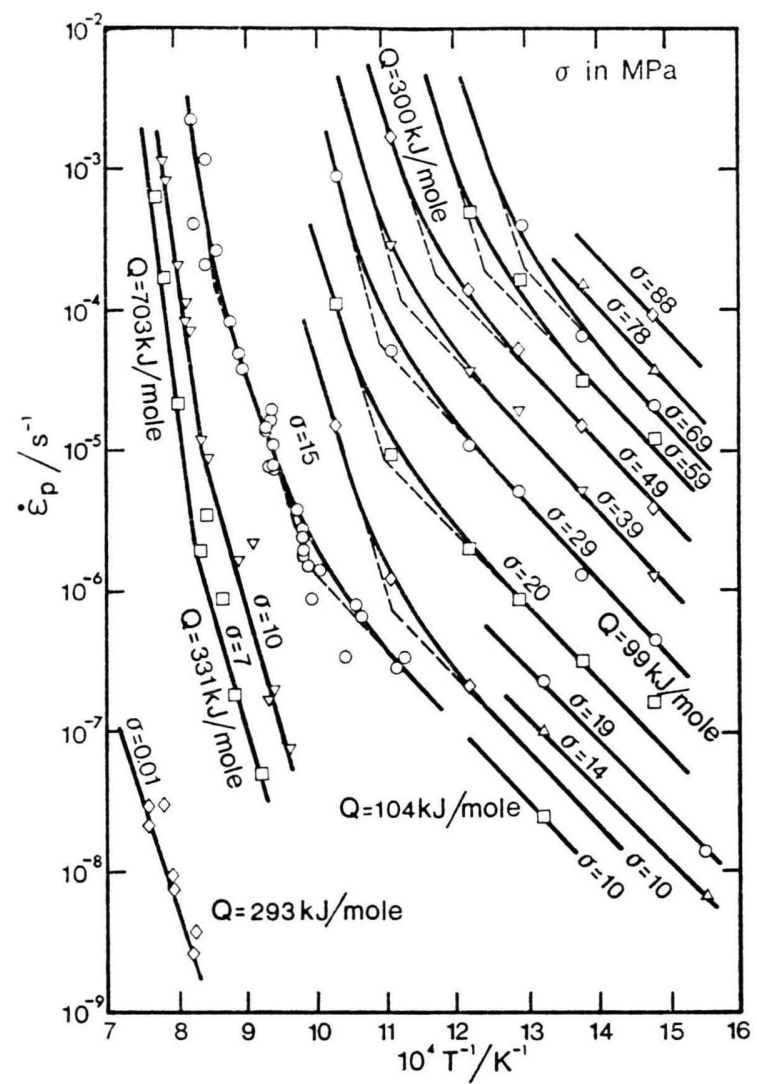

Fig. 9. The minimum creep rate of copper [32].

"creep of copper is a multistep reaction composed of at least two and possibly three distinct steps acting in parallel". The temperature dependence of the strain rate in copper is shown in Figure 9. $\mathrm{Li}$ [22] has listed a number of materials for which the plastic flow is controlled by the simultaneous operation of various mechanisms.

\section{Conclusion}

Deformation behavior is often controlled by more than one mechanism and the experimental results have to be analyzed accordingly. The present study, based on a rigorous theory, indicates the various behaviors that are associated with the various types of combinations. It has to be recognized that the establishment of a particular kinetics is not an easy undertaking: experimental results have to be obtained in wide stress and temperature ranges for almost the same state of the material, and this is not always possible. Then, the experimental results must be fitted to one of the proposed Eq. (9) to (11) or to the combinations of them, utilizing the method of Hanley and Krausz [33]. It is hoped that more investigators will follow the rigorous method of kinetics analysis and thus contribute to the understanding of deformation behavior.

\section{Acknowledgements}

The financial assistance provided by the Natural Sciences and Engineering Research Council of Canada is gratefully acknowledged.

\section{Appendix}

The steady state net flow over each of the $n$ barriers of the consecutive system shown in Fig. 2 is obtained $[8,20]$ as

$$
\begin{aligned}
& F_{1}=\varrho_{11} \mathrm{k}^{1}-\varrho_{2}{ }^{1} \mathrm{k}_{2}=F, \\
& \vdots \\
& F_{i}=\varrho_{i i} \mathrm{k}^{i}-\varrho_{i+1}{ }^{i} \mathrm{k}_{i+1}=F, \\
& \vdots \\
& F_{n}=\varrho_{n} \mathrm{k}^{n}-\varrho_{1}{ }^{n} \mathrm{k}_{1}=F,
\end{aligned}
$$

where $\varrho_{i}$ is the concentration of dislocations (or flow units) in the $i$-th valley, and the activation rates are expressed for forward activation as $[8,9]$

$$
\begin{aligned}
{ }_{i} \mathrm{k}^{i} & =\frac{k T}{h} \exp \left(-\frac{{ }_{i} \Delta G^{i}}{k T}\right) \\
& =\frac{k T}{h} \exp \left[-\frac{i \Delta G^{\neq i}-{ }_{i} W^{i}(\tau)}{k T}\right]
\end{aligned}
$$

and for backward activation as

$$
\begin{aligned}
{ }^{i} \mathrm{k}_{i+1} & =\frac{k T}{h} \exp \left(-\frac{i \Delta G_{i+1}}{k T}\right) \\
& =\frac{k T}{h} \exp \left[-\frac{i \Delta G_{i+1}^{\ddagger}+{ }^{i} W_{i+1}(\tau)}{k T}\right] .
\end{aligned}
$$

Multiplying the $i$-th line $(i=2, \ldots, n)$ of Eq. (A.1) by

$$
\underset{j=2}{i} \frac{j-1 \mathrm{k}_{j}}{j \mathrm{k}^{j}}
$$

and adding we get

$$
\begin{aligned}
& \varrho_{1}\left({ }_{1} \mathrm{k}^{1}-\frac{{ }^{1} \mathrm{k}_{2}{ }^{2} \mathrm{k}_{3} \ldots{ }^{n-1} \mathrm{k}_{n}{ }^{n} \mathrm{k}_{1}}{{ }_{2} \mathrm{k}^{2}{ }_{3} \mathrm{k}^{3} \ldots n \mathrm{k}^{n}}\right) \\
& =F\left[1+\sum_{i=2}^{n} \underset{j=2}{i} \frac{{ }^{j-1} \mathrm{k}_{j}}{{ }_{j} \mathrm{k}^{j}}\right]
\end{aligned}
$$


or, dividing by ${ }_{1} k^{1}$

$\varrho_{1}\left[1-\exp \left({ }_{1} W_{1} / k T\right)\right]=F \sum_{i=1}^{n} \frac{1}{{ }_{1} k^{i}}=F \sum_{i=1}^{n} t_{1 i}$ (

Equation (A.2) is obtained by considering that

$$
\begin{aligned}
{ }_{1} \mathrm{k}_{2}{ }_{2} \mathrm{k}_{3} \ldots{ }^{n-1} \mathrm{k}_{n}{ }^{n} \mathrm{k}_{1} \\
\hline{ }_{1} \mathrm{k}^{1}{ }_{2} \mathrm{k}^{2} \cdots{ }_{n} \mathrm{k}^{n} \\
=\exp \left[{ }_{1} \Delta G^{1}-1 \Delta G_{2}+{ }_{2} \Delta G^{2}-\cdots\right. \\
\left.\left.\quad+{ }_{n} \Delta G^{n}-{ }^{n} \Delta G_{1}\right) / k T\right] \\
=\exp \left[{ }_{1} \Delta G^{1 \neq}-1 \Delta G_{2}^{\neq}+{ }_{2} \Delta G^{2 \neq}-\cdots\right. \\
\left.\left.\quad+{ }_{n} \Delta G^{n \neq}-{ }^{n} \Delta G_{1}^{\neq}\right) / k T\right] \\
\quad \times \exp \left[-\left({ }_{1} W^{1}+{ }^{1} W_{2}+{ }_{2} W^{2}+\cdots\right.\right. \\
\left.\left.\quad+{ }_{n} W^{n}+{ }^{n} W_{1}\right) / k T\right] \\
=\exp \left[-\left({ }_{1} W^{1}+{ }^{1} W_{2}+\cdots+{ }^{n} W_{1}\right) / k T\right] \\
=\exp \left(-{ }_{1} W_{1} / k T\right)
\end{aligned}
$$

as illustrated in Fig. 2, and because after overcoming the $n$-barriers of the consecutive system at zero stress level the dislocations are in an identical energy state so that

$$
\begin{aligned}
{ }_{1} \Delta G^{1 \neq}+{ }_{2} \Delta G^{2 \neq}+\cdots+{ }_{n} \Delta G^{n \neq} & \\
& =1 \Delta G_{2}^{\neq}+{ }^{2} \Delta G_{3}^{\neq}+\cdots+{ }^{n} \Delta G_{1}^{\neq} .
\end{aligned}
$$

Also

$$
\begin{aligned}
& \frac{1}{{ }_{1} \mathrm{k}^{1}} \underset{j=2}{i} \frac{{ }^{j-1} \mathrm{k}_{j}}{{ }_{j} \mathrm{k}^{j}}=\frac{1 \mathrm{k}_{2} \ldots{ }^{i-1} \mathrm{k}_{i}}{{ }_{1 \mathrm{k}^{1}{ }_{2} \mathrm{k}^{2} \cdots i \mathrm{k}^{i}}=\frac{h}{k T}} \\
& \cdot \exp \left[\frac{1 \Delta G^{1}-1 \Delta G_{2}+{ }_{2} \Delta G^{2}-\cdots-i-1 \Delta G_{i}+{ }_{i} \Delta G^{i}}{k T}\right] \\
& =\frac{h}{k T} \exp \left(\frac{1 \Delta G^{i}}{k T}\right)=t_{1 i}=\frac{1}{{ }_{1} \mathrm{k}^{i}}
\end{aligned}
$$

where ${ }_{1} \Delta G^{i}$ is illustrated in Fig. 2, and ${ }_{1} \mathrm{k}^{i}$ represents the rate of activation for a dislocation from the first valley over the $i$-th barrier in the forward direction.

Because the choice of the first barrier is arbitrary, $n$ equations similar to Eq. (A.2) may be written as

$$
\varrho_{i}\left[1-\exp \left(-{ }_{i} W_{i} / k T\right)\right]=F \sum_{j=i}^{n+i-1} t_{i j}
$$

where ${ }_{i} W_{i}$, as ${ }_{1} W_{1}$, is the work done by the stress as a dislocation moves over the whole system, and

$$
{ }_{i} W_{i}={ }_{1} W_{1}={ }_{2} W_{2}=\cdots={ }_{n} W_{n} .
$$

[1] J. C. M. Li in "Dislocation Dynamics", edited by A. R. Rosenfield, G. T. Hahn, A. L. Bement, Jr., R. I. Jaffee, McGraw-Hill, New York 1968, p. 87.

[2] T. Surek, M. J. Luton, and J. J. Jonas, Philos. Mag. 27,425 (1973).

[3] M. F. Ashby, Acta Met. 20, 887 (1972).
Adding the $n$ equations, one obtains

$$
F=\varrho_{t} \frac{1-\exp \left(-{ }_{1} W_{1} / k T\right)}{\sum_{i=1}^{n}\left(\sum_{j=i}^{n+i-1} t_{i j}\right)}
$$

where the total number of dislocations is

$$
\varrho_{t}=\varrho_{1}+\varrho_{2}+\cdots+\varrho_{n} .
$$

Considering that after activation over the $i$-th barrier a dislocation produces the strain $\delta_{i}$, the strain rate resulting from the flow of the $\varrho_{t}$ dislocations over the consecutive system is

$$
\dot{\varepsilon}_{\mathrm{p}}=\Sigma_{i} \delta_{i} F_{i}=F \Sigma_{i} \delta_{i}=F \delta
$$

and utilizing the notation

$$
\varepsilon_{0}=\delta \varrho_{t},
$$

Equation (7) is obtained:

$$
\dot{\varepsilon}_{\mathrm{p}}=\varepsilon_{0} \frac{1-\exp \left(-{ }_{1} W_{1} / k T\right)}{\sum_{i=1}^{n}\left(\sum_{j=i}^{i+n-1} t_{i j}\right)} .
$$

The dislocation density in front of each barrier, as given by Eq. (8), is obtained by combining (A.3) and (A.4). For a two barrier system, (7) becomes

$$
\dot{\varepsilon}_{\mathrm{p}}=\varepsilon_{0} \frac{1-\exp \left(-{ }_{1} W_{1} / k T\right)}{\frac{1}{{ }_{1} \mathrm{k}^{1}}+\frac{1}{{ }_{2} \mathrm{k}^{2}}+\frac{1}{{ }_{1} \mathrm{k}^{2}}+\frac{1}{{ }_{2} \mathrm{k}^{1}}} .
$$

In a comprehensive study of these systems [23], it was shown that only three of the four terms in the denominator may have an individually noticeable effect. Furthermore, neglecting the exponential term in the numerator, and when only two terms in the denominator are noticeable, the strain rate is expressed as

$$
\dot{\varepsilon}_{\mathrm{p}}=\frac{\varepsilon_{0}}{\frac{1}{\mathrm{k}_{1}}+\frac{1}{\mathrm{k}_{2}}}=\frac{\dot{\varepsilon}_{0}}{\exp \left(\frac{\Delta G_{1}}{k T}\right)+\exp \left(\frac{\Delta G_{2}}{k T}\right)}
$$

where $\dot{\varepsilon}_{0}$ has been used for $\frac{k T}{h} \varepsilon_{0}$. Hence, Eq. (11) is obtained.

[4] J. C. M. Li, Trans. Metall. Soc. AIME 233, 219 (1965).

[5] J. J. Gilman, Micromechanics of Flow in Solids, McGraw-Hill, New York 1969, p. 201.

[6] J. P. Hirth, in "Inelastic Behavior of Solids", edited by M. F. Kanninen, W. F. Adler, A. R. Rosenfield, and R. I. Jaffee, McGraw-Hill, New York 1970, p. 281. 
[7] A. S. Krausz and H. Eyring, J. Appl. Phys. 42, 2382 (1971).

[8] A.S. Krausz and H. Eyring, "Deformation Kinetics", Wiley-Intersci. New York 1975.

[9] S. Glasstone, K. J. Laidler, and H. Eyring, The Theory of Rate Processes, McGraw-Hill, New York 1941.

[10] C. N. Hinshelwood, The Structure of Physical Chemistry, Clarendon Press, Oxford 1952.

[11] S. W. Benson, Foundations of Chemical Kinetics, McGraw-Hill, New York 1960.

[12] T. Ree and H. Eyring, in "Rheology Theory and Applications", edited by F. R. Eirich, Academic Press, New York 1958, p. 83.

[13] V. Celli, M. Kabler, T. Ninomiya, and R. Thomson, Phy. Rev. 131, 58 (1963).

[14] T. O'D. Hanley, A. S. Krausz, and N. Krishna, J. Appl. Phys. 45, 2016 (1974).

[15] A. S. Krausz and B. Faucher, Scripta Met. 13, 91 (1979).

[16] J. P. Hirth and J. Lothe, Can. J. Phys. 45, 809 (1967).

[17] A. S. Krausz, Acta Met. 16, 897 (1968).

[18] A. S. Krausz, Mater. Sci. Eng. 6, 260 (1970).

[19] U. F. Kocks, in "Physics of Strength and Plasticity", edited by A. S. Argon, MIT Press, Cambridge Mass., 1969 , p. 143.
[20] A. S. Krausz, Mater. Sci. Eng. 26, 65 (1976).

[21] R. C. Gifkins, J. Mater. Sci. 5, 156 (1970).

[22] J. C. M. Li, in "Rate Processes in Plastic Deformation of Materials", edited by J. C. M. Li and A. K. Mukherjee, ASM, Cleveland, 1975, p. 479.

[23] B. Faucher, and A. S. Krausz, J. Appl. Phys. 49, 3946 (1978).

[24] G. Alefeld, Z. Naturforsch. 17 a, 899 (1962).

[25] T. G. Langdon and F. A. Mohamed, J. Aust. Int. Metals 22, 189 (1977).

[26] C. C. Law and D. N. Beshers, Scripta Met. 6, 635 (1972).

[27] D. J. Quesnel, A. Sato, and M. Meshii, Mater. Sci. Eng. 18, 208 (1975).

[28] D. Tseng and K. Tangri, Scripta Met. 11, 719 (1977).

[29] E. Kuramoto, Y. Aono, and K. Kitajima, Scripta Met. 13, 1039 (1979).

[30] R. J. Arsenault, Philos. Mag. 26, 1481 (1972).

[31] B. Burton and G. L. Reynolds, Acta Met. 21, 1073 (1973).

[32] E. R. Gilbert and D. E. Munson, Trans. Metall. Soc. AIME 233, 429 (1965).

[33] T. O'D. Hanley and A. S. Krausz, J. Appl. Phys. 45, 2013 (1974). 